\title{
Visual noise reveals category representations
}

\author{
JASON M. GOLD \\ Indiana University, Bloomington, Indiana \\ ANDREW L. COHEN \\ University of Massachusetts, Amherst, Massachusetts \\ and \\ RICHARD SHIFFRIN \\ Indiana University, Bloomington, Indiana
}

\begin{abstract}
How are categories represented in human memory? Exemplar models assume that a category is represented by individual instances from that category that have been experienced. More generally, a category might be represented by multiple templates stored in memory. A new item is classified according to its similarity to these templates. Prototype models represent a category with a single summary abstraction (i.e., a single template), often the central tendency of the experienced items. A new item is classified according to its similarity to these category prototypes. Here, we show how a technique for correlating observers' responses with external noise can be used not only to distinguish single- from multiple-template representations, but also to induce the form of these templates. The technique is applied to two tasks requiring categorization of simple visual patterns; the results demonstrate that observers used multiple traces to represent their categories, and thus highlight the procedure's potential for use in more complex settings.
\end{abstract}

A central problem in cognitive psychology is how we represent perceptual categories in memory and the processes by which these representations are used to classify new perceptual inputs. For example, what memory representations and processes do we use to classify an unknown person as a human, rather than as a toaster or a frog? Exemplar models assume that people represent categories on the basis of stored traces of individual category instances that they have experienced (Medin \& Schaffer, 1978; Nosofsky, 1986). A generalized form of this approach would represent a category on the basis of multiple templates, although not necessarily via a one-to-one mapping with specific instances. A new item is classified according to how closely it resembles the stored traces from one category in comparison with the stored traces from alternative categories (Nosofsky, 1986). Prototype models assume that people form, for each category, a single summary representation (or single template) which is often assumed to be the central tendency across all category instances. A new item is classified on the basis of its similarity to each category prototype (Minda \& Smith, 2001, 2002). These model distinctions have arisen in category

This research was supported by NEI Grant 1 R03 EY015787 to J.M.G. and NIMH Grants 1 R01 MH12717 and 1 R01 MH63993 to R.S. J.M.G. and R.S. are affiliated with the departments of psychology and cognitive science at Indiana University. Correspondence concerning this article should be sent to J. M. Gold, Departments of Psychology and Cognitive Science, Indiana University, 1101 East 10th Street, Bloomington, Indiana 47405 (e-mail: jgold@indiana.edu). domains ranging from high-level concepts to low-level perception, with the latter domain often being modeled with Bayesian pattern recognition approaches (Duda \& Hart, 1973; Graham, 1989; Knill \& Richards, 1996).

Such Bayesian template-matching models often incorporate the strong assumption that category decisions are based on the statistically optimal use of information (Green \& Swets, 1966; Tjan, Braje, Legge, \& Kersten, 1995). For example, consider the simple visual pattern categorization task depicted in Figure 1. The observer is shown a white square (the signal) at one of four possible locations, randomly selected on each trial. Each of the four locations where the square could appear is divided into a $4 \times 4$ grid, and Gaussian noise is added to each section within each of these four grids (see Figure 1 for more stimulus details). The observer's task is to indicate whether the white square signal appears above or below fixation. Thus, in this very simple categorization task, there are two categories (top and bottom), each with two members (left and right). The contrast of the white square signal is placed at a level where performance is at threshold (i.e., $71 \%$ correct).

It turns out that the optimal strategy for this task is to represent each of the two categories by two templates and then classify test stimuli according to their similarity to the templates using a decision rule that is remarkably similar to the decision rule used by several exemplar-based categorization models (Nosofsky, 1990). In particular, the ideal category templates are the four noise-free versions of the stimuli shown in Figure 1, a white square plus three gray squares. The relative likelihood of the top and bot- 

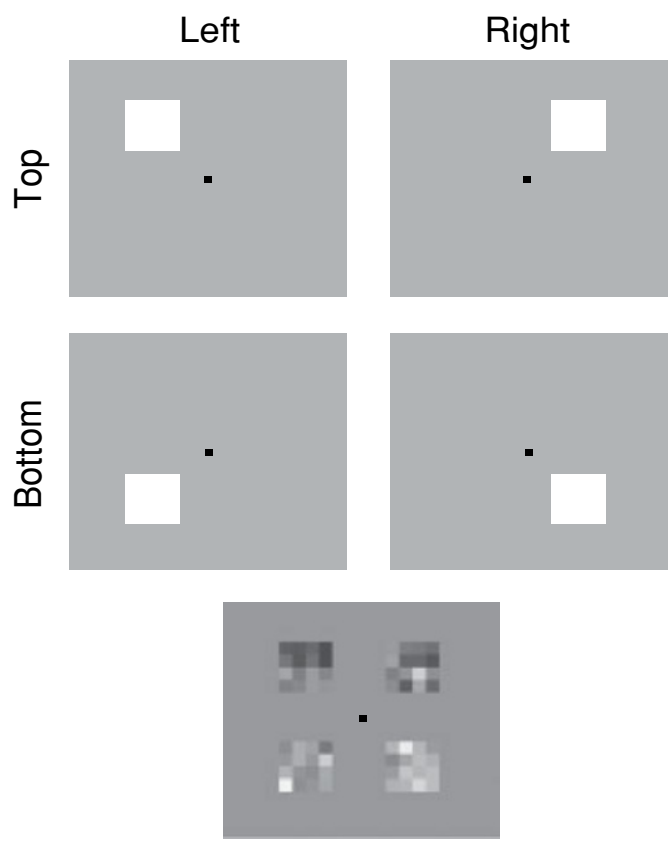

Figure 1. Stimuli used in the square categorization task. The top four images show the noise-free versions of the square stimuli (signals) from each category (top and bottom). The bottom image shows an example of a noisy bottom right stimulus. Each square subtended $0.65^{\circ}$ of visual angle from a viewing distance of $130 \mathrm{~cm}$. The distance of the center of each square from the center of the display was $1.39^{\circ}$. Each square was coarsely divided into a $4 \times 4$ grid of pixels. The screen pixels within each grid location were set to the same contrast value (with contrast of a pixel being defined as the difference between pixel and background luminance, normalized by background luminance). A $4 \times 4$ screen pixel fixation point remained at the center of the display for the duration of the experiment. On each trial, the stimulus was shown for approximately $500 \mathrm{msec}$. The background luminance was $81.4 \mathrm{~cd} / \mathrm{m}^{2}$. The contrast variance of the Gaussian noise added to the square grids was .04. Accuracy feedback was provided in the form of a high or low beep.

tom categories in the presence of a noisy test stimulus, $S$ (signal and noise at all locations), is given by

$$
\frac{L(\text { Top } \mid S)}{L(\text { Bottom } \mid S)}=\frac{\sum_{t \in \text { Top }} \exp \left[-\frac{1}{2 \sigma^{2}} \sum_{p}\left(S_{p}-T_{t p}\right)^{2}\right]}{\sum_{b \in \text { Bottom }} \exp \left[-\frac{1}{2 \sigma^{2}} \sum_{p}\left(S_{p}-T_{b p}\right)^{2}\right]}
$$

where $t$ and $b$ range over the templates from the top and bottom categories, respectively; $p$ ranges over the $8 \times 8$ grid of pixels that defines the set of potential stimulus locations; $S_{p}$ is the $p$ th pixel of stimulus $S ; T_{x p}$ is the $p$ th pixel from the template for category member $x$; and $\sigma$ is the standard deviation of the externally added noise (Green \& Swets, 1966; Tjan et al., 1995). Category top is selected if the likelihood ratio is greater than 1; bottom is selected if the ratio is less than 1 . We term this a multiple-template model rather than an exemplar model because a "pure" exemplar model would represent a category (e.g., top) on the basis of each and every signal-plus-noise presentation that was accompanied by feedback. Using a multipletemplate model as a stand-in for an exemplar model seems reasonable for tasks such as the present one, in which the target signals are known exactly and the storage of exact noise patterns is implausible given known limitations on human memory.

An alternative, suboptimal model that is akin to a prototype representation can be posited. In such a model, each category would be represented by a single template $T$, consisting of two light squares above and two gray squares below (for top) or two light squares below and two gray squares above (for bottom). The relative likelihood of the top and bottom categories given a test stimulus $S$ is

$$
\frac{L(\text { Top } \mid S)}{L(\text { Bottom } \mid S)}=\frac{\exp \left[-\frac{\sigma^{2}}{2} \sum_{p}\left(S_{p}-T_{T p}\right)^{2}\right]}{\exp \left[-\frac{\sigma^{2}}{2} \sum_{p}\left(S_{p}-T_{B p}\right)^{2}\right]},
$$

where $T_{T}$ and $T_{B}$ are the single-prototype templates for categories top and bottom, respectively.

Because only Equation 1 involves a sum of exponentials, these single- and multiple-template models differ in the way they incorporate nonlinearities. It is easy to see that a logarithmic transformation reduces Equation 2 to a simple ratio of distances between the stimulus and each of the prototypes, but this simplification is not possible when a log of a sum is taken, as in Equation 1. This seemingly small distinction between the models is the key to the technique for discriminating between single- and multiple-template category representations described in this article. We shall analyze the formation of classification images, which is an increasingly common approach for estimating the templates used by an observer (Ahumada, 2002; Ahumada \& Lovell, 1971). This technique, known as reverse correlation (Ringach, Sapiro, \& Shapley, 1997) or response classification (Beard \& Ahumada, 1998), involves computing the correlation between the noise that is added to each pixel in the stimulus and the observer's decisions across trials. It has been used to estimate observer templates in a wide variety of psychophysical tasks, ranging from simple detection of gratings (Ahumada \& Beard, 1999) to face and object recognition (Gold, Murray, Bennett, \& Sekuler, 2000; Sekuler, Gaspar, Gold, \& Bennett, 2004).

In the present procedure, the exact noises that had been added to the signal on each trial are sorted into separate bins (only the noises are classified; the signal is discarded). In our extension of this procedure, there is one bin for each combination of signal presented and response given, and the noise patterns within each bin are averaged. In the case of the square categorization task described in Figure 1, there are four possible signals (top left, top right, bottom left, and bottom right) and two possible responses ("top" and "bottom"), so the method produces eight average noise patterns, called classification images. A classification image shows the relative weighting given to each pixel by the observer for 
a particular signal-response combination over the course of the experiment (Ahumada, 2002).

We first used simulations to verify the intuition that single- and multiple-template models produce distinctly and measurably different patterns of results when the external noise is analyzed in this fashion. For the top-bottom classification experiment described above, we used Equations 1 and 2 to classify 16,000 signal-plus-noise trials. In the multiple-template simulation, we used the ideal templates - that is, the four noise-free signals shown in Figure 1. The templates used in the single-template model simulation were combined versions of the two signals within each category: The single top template was composed of two light top squares (with two gray bottom squares), and the single bottom template was composed of two light bottom squares (with gray top squares).

The eight classification images that were produced by averaging the noise patterns in each signal-response bin are shown for the single-template (prototype) model in Figure 2A and for the multiple-template (exemplar) model in Figure 2B. To understand these plots, consider the four small squares in the top left bin of the upper panel of Figure 2A. These squares show the correlation between the externally added noise and the prototype observer's re- sponses at each pixel in each square location for the trials where the stimulus was top left and the observer responded "top." First, notice that the top two squares are lighter than the background, whereas the bottom two squares are darker than the background. Second, note that the pattern was reversed when the observer responded "bottom." Whereas lighter noise in the top regions and darker noise in the bottom regions made the observer more likely to respond "top," the opposite pattern made the observer more likely to respond "bottom." The key finding, however, is that the placement of the signal in the left or right position did not alter the classification images, which remained the same for both signal-left and signal-right placements. This is not the case, however, for the multiple-template model shown in Figure 2B. Light squares above and dark squares below still led the multiple-template observer to respond "top," and vice versa; however, the classification images differed depending on whether the signal was presented on the left or the right.

Consider the case where the signal is top left (i.e., the top left bins in Figures 2A and 2B). For the prototype observer, the top locations for squares are weighted equally, as they are when the signal is top right. However, the multiple-template (exemplar) observer shows a greater

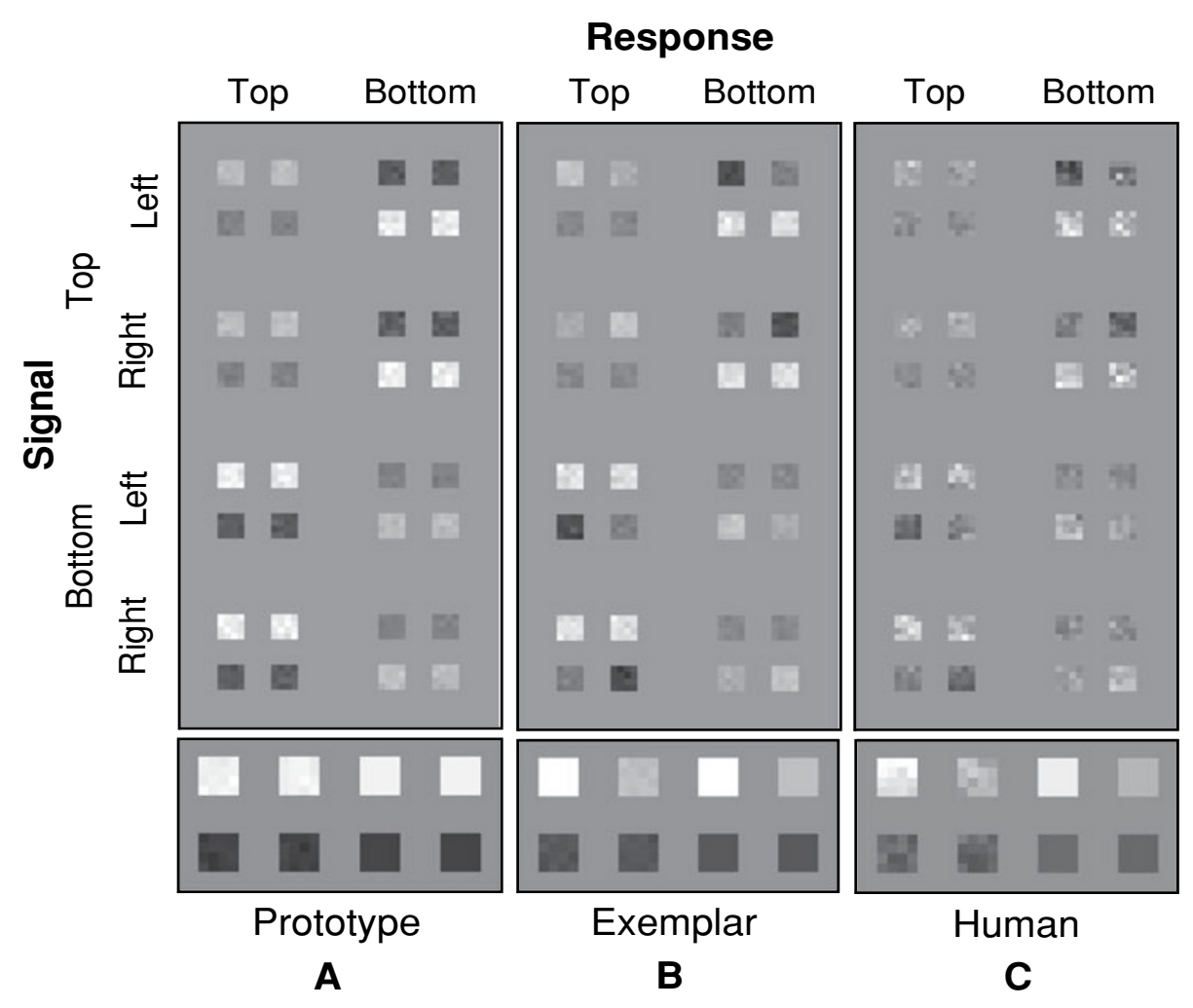

Figure 2. Response classification results in the square categorization task for (A) a prototype model observer, (B) an exemplar model observer, and (C) human observers. Each block of four squares in the top row shows the classification images resulting from the corresponding signal-response combination. The separate row of images at the bottom of the figure summarizes the data for each observer by collapsing across all of the bins in the image above (left side) and smoothing the data (right side) (see text for details). 
influence of lightness in the top left location when the signal is present in the top left (Row 1) and a greater influence of lightness in the top right location when the signal is present in the top right (Row 2). Notice that this kind of asymmetry is present in all of the other signal-response bins for the multiple-template observer; it is always the case that the location where the signal is actually present is weighted more than the adjacent location within the same category. This difference is made even more apparent in lower panels of Figures $2 \mathrm{~A}$ and $2 \mathrm{~B}$, which summarize the data for each observer by collapsing across all of the bins. These summaries were computed by flipping and/or contrast reversing each bin to make it consistent with the "signal $=$ top left $/$ response $=$ top" bin (e.g., the "signal $=$ top left / response = bottom" bin was contrast reversed, and the "signal = top right / response = top" bin was flipped about the vertical midline). The bin (four squares) farthest to the left in each summary figure (three rectangles at the bottom of Figure 2) is the raw summary data computed by simply collapsing across bins as described above. The bin farthest to the right in each summary figure is a smoothed version of the raw summary figure, computed by replacing all of the values within each square region with the mean value across pixels.

These simulations show that, in contrast to a prototype observer, a multiple-template observer gives more weight to the location where the signal is present on a trial. This difference between models is caused by the exponential nonlinearities in Equations 1 and 2 that apply singly for the prototype model (Equation 2) and summed for the multipletemplate model (Equation 1). To aid intuition, consider that for the multiple-template model, an incorrect classification requires a great deal of noise that is negatively correlated with the signal to overcome the signal that is present, an effect that is magnified by the nonlinearity in the models. The prototype model does not sum the exponentiated locations separately, so noise in either location has an equal effect. It is important to note that this difference between the models is independent of the specific choice of prototype and depends only on the use of a single-template-per-response category.

We next applied this same analysis to the classification data for 4 human observers in the same experiment. Each human observer participated in 4,000 trials. The results of this analysis for the data combined across all 4 observers are shown in Figure 2C (the individual observer patterns were similar to those shown in Figure 2C, but noisier). These images were computed from the human data in the same fashion as the images in Figures 2A and 2B. The data clearly show that human observers exhibited the same differential pattern of stimulus location weighting as the multiple-template model observer (Figure 2B), demonstrating that the pure prototype model is not adequate: An adequate model must include more than one template per category. We quantified this effect by computing the ratio of the top locations farthest to the left and farthest to the right in each of the smoothed summary plots for the simulated and human observers. The results of this analysis are shown in Figure 3. As expected, the correlation ratio for the proto-

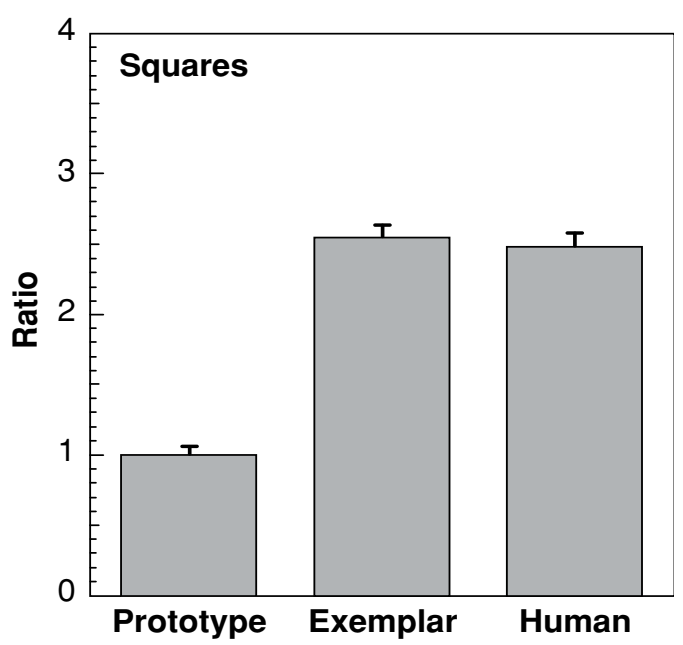

Figure 3. Ratios of the mean values obtained for the left and right top square locations in the smoothed classification images shown at the bottom of Figure 2. Error bars correspond to \pm 2 $S D$.

type observer was exactly 1 , indicating that the weighting of the two locations was the same for this observer. In contrast, the correlation ratios for the exemplar and human observers were much greater than $1(\sim 2.5)$ and were nearly identical.

Note that the true model used by human observers might well be a complex mixture of multiple-template representations and decision rules, rather than either of the pure models we have simulated here. Finding the true model used by our observers, or testing which ideal model better approximated the true model, was not the aim of this article. Nonetheless, we carried out a number of comparison analyses to see which ideal model best predicted the trial-by-trial responses made by the human observers. In all cases, the multiple-template model fared better than the single-template model. The strongest test (and the simplest to understand) involved looking at the trials on which the two models strongly predicted opposite responses among the 4,000 experienced by each observer; these are the most diagnostic trials. Because the two models generally make highly correlated predictions, such diagnostic trials are relatively rare, occurring on only 135 trials across the data for all 4 human observers. However, on these highly diagnostic trials, the human observers' responses matched the predictions of the "ideal" multipletemplate model on 113 of the 135 trials.

Thus, our results provide an important proof of concept-namely, that the response classification technique can be used to discriminate between single- and multiple-template models of categorization. To the extent that the multiple-template model is a reasonable approximation of the exemplar model, this technique has potentially wide-ranging applications for distinguishing prototype and exemplar theories of perceptual categorization.

The four-square task, however, is admittedly simple. To test the generalizability of our results, we applied this technique to a task that requires classifications along di- 
mensions that are more abstract than the spatial position of the stimuli. Figures $4 \mathrm{~A}-4 \mathrm{C}$ illustrate a task in which observers had to classify patterns on the basis of their spatial frequency (i.e., bar width) while the stimuli varied in both frequency and orientation. In this task, there were two categories: high frequency ( 5 cycles per degree; left panel of Figure 4A) and low frequency ( 2 cycles per degree; right panel of Figure 4A). Within each category, one grating is oriented $45^{\circ}$ left of vertical (bottom row of Figure $4 \mathrm{~A}$ ) and the other is oriented $45^{\circ}$ right of vertical (top row of Figure 4A). As in the square categorization task, the stimulus was corrupted by white Gaussian pixel noise (e.g., the top of Figure 4C), and the observer's task was to classify a stimulus as belonging to one of the two possible categories (high or low frequency; see Figure 4 for more stimulus details).

Figure 4B represents in Fourier frequency space the stimuli shown in Figure 4A. As illustrated by the bottom of
Figure 4C, spatial frequency in these plots is represented as the distance from the center of the image, and orientation is represented as the angle made relative to the horizontal axis. The amount of relative power at each constituent frequency component is represented by the contrast at each location in each plot. For clarity, only frequencies below 8 cycles per image are shown in the figures. Figure 4B shows that the stimuli in Figure 4A can be equivalently represented as localized "bumps" in Fourier space. In addition, because white Gaussian contrast noise in the spatial domain introduces white Gaussian amplitude noise in the spatial frequency domain, our response classification analyses can be equivalently carried out in Fourier space (Ahumada, Marken, \& Sandusky, 1975).

Despite the differences in stimuli, the multipletemplate (exemplar) and single-template (prototype) model decision rules are the same in both the grating and square discrimination tasks. Just as in the square categori-

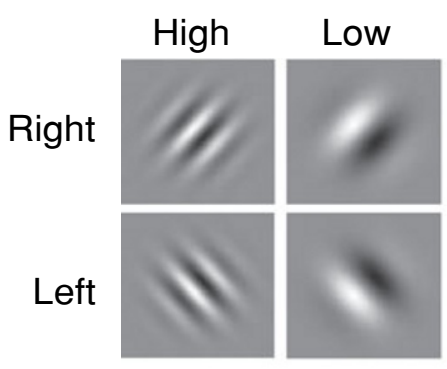

A

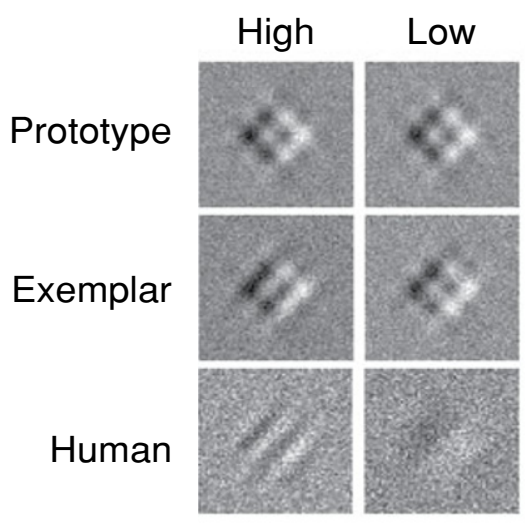

D

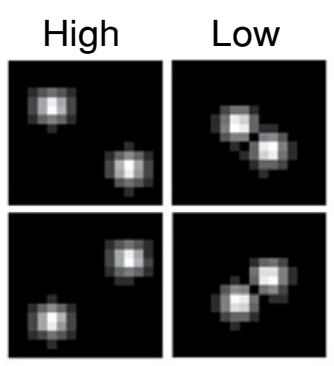

B

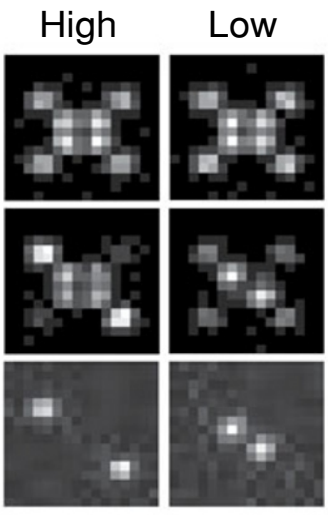

E

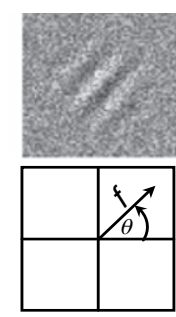

C

Figure 4. (A) Signals used in the Gabor categorization task. (B) The same signals represented in Fourier space (amplitude spectra only). Each plot shows frequencies less than or equal to 8 cycles per image. (C) Top: Example of a noisy, high frequency, right-oriented Gabor stimulus in the spatial domain. Bottom: A description of how frequency $f$ and orientation $\theta$ are represented in Figure 4B. The Gabor stimuli were $64 \times 64$ pixels in size, subtending $1.05^{\circ}$ of visual angle from a viewing distance of $130 \mathrm{~cm}$. Fixation was maintained by a dark box that surrounded the stimulus region for the duration of the experiment. The spatial frequencies of the Gabors were 5 and 2 cycles per degree of visual angle, and the orientations were $\pm 45^{\circ}$ to the left and right of vertical. The stimulus duration was approximately $500 \mathrm{msec}$, and the Gaussian noise added to each pixel had a contrast variance of .04. Accuracy feedback was provided in the form of a high or low beep. (D) Summary spatial classification images for both the model observers and the human observers in each category (high and low frequency). (E) Frequency-space summary classification images for both the model observers and the human observers in each category. (F) Ratios of cross-correlation values obtained for each observer type in each condition (see text for details). Error bars correspond to $\pm 2 S D$. 
zation task, we would expect the multiple-template model to produce different classification images depending on the within-category signal presented - that is, there should be differences dependent on signal orientation. Figure 4D (spatial domain) and Figure 4E (spatial frequency domain) show the results of this experiment. The top row gives the simulated results for the single-template (prototype) model and the second row for the multiple-template (exemplar) model, each based on 45,000 simulated trials. The bottom row gives the combined results for 3 human observers (each participated in 15,000 trials, the greater number of trials being necessary because of the greater number of pixels in the stimuli).

As in the summary plots described at the bottom row of Figure 2, the symmetries across the various signalresponse bins allowed us to produce a single summary image for each spatial frequency response type (i.e., one image for high frequency and one image for low frequency). This figure shows that the prototype model produced equal classification images when presented with stimuli with different orientations, whereas the multiple-template model and human observers showed significant orientationdependent differences in the classification images. We quantified this effect by computing the ratio of the similarities (cross-correlations) of the Fourier signal plots shown in Figure 4B within a given category (high or low) to the corresponding Fourier classification plots shown in Figure 4E. The results of this analysis are shown in Figure 4F. As with the square categorization task, the performance of the exemplar model matched the performance of the human observers far more closely than the performance of the prototype model did. Thus, we can conclude that any adequate model of human performance in this task must include multiple templates.

As with the square categorization task, we carried out a series of tests to determine which ideal model better predicted the trial-by-trial responses made by the human observers. Unlike the results for the square categorization task, the results of the analysis of the multiple-template model were only slightly better than the results for the single-template model. Furthermore, it is clear from the data that the idealized multiple template model described above does not fully capture the pattern of human data. In particular, as can be seen in Figures 4D-4E, the multipletemplate model predicts a much wider range of frequency and orientation influence than the human data show. These results raise an important cautionary note but also highlight a benefit of the present technique: Although our data do not allow us to distinguish between the idealized single- and multiple-template models, the response classification technique can still be used to make inferences about the form of the templates used by observers, as well as to demonstrate that multiple templates must be a part of any adequate model. It is also worth noting that, although both of the examples presented here involved adding noise at the level of individual pixels, it is possible to restrict the added noise to a stimulus subspace (Ringach et al., 1997) and to add noise along higher order stimulus dimensions, such as size, curvature, and aspect ratio (e.g.,
Neri, Parker, \& Blakemore, 1999). Adding noise along these kinds of higher order dimensions could greatly reduce the size of stimulus perturbation space and may be better suited to more standard categorization tasks (see Nosofsky, 1986).

In sum, our results demonstrate that the response classification technique is an effective tool for making inferences about the number and form of templates used to make category judgments, for assessing the adequacy of single-template (prototype) models, and equivalently, for demonstrating the existence of multiple templates within each category. Our two experimental demonstrations involved relatively simple tasks, but they each required quite different visual representations. However, it is worth emphasizing that the experiments presented here were explicitly designed to be simple and easy to analyze and to provide a straightforward proof of concept. Although the results of our experiments happen to support an exemplartype representation, they in no way offer a systematic and thorough comparison of prototype and exemplar models. In future research, we hope to use the technique described here to explore the kinds of representations human observers use in more complex and realistic categorization tasks, especially ones in which there are many more items within each category and the templates of the alternative models are not obvious a priori.

\section{REFERENCES}

Ahumada, A. J., JR. (2002). Classification image weights and internal noise level estimation. Journal of Vision, 2, 121-131.

Ahumada, A. J., JR., \& BeArd, B. L. (1999). Classification images for detection [Abstract]. Investigative Ophthalmology \& Visual Science, 40, S572.

Ahumada, A. [J., JR.], \& Lovell, J. (1971). Stimulus features in signal detection. Journal of the Acoustical Society of America, 49, 17511756.

Ahumada, A. [J.], JR., Marken, R., \& Sandusky, A. (1975). Time and frequency analyses of auditory signal detection. Journal of the Acoustical Society of America, 57, 385-390.

Beard, B. L., \& Ahumada, A. J., JR. (1998, January). Technique to extract relevant image features for visual tasks. Paper presented at the International Society for Optical Engineering conference, San Jose, CA.

DudA, R. O., \& HART, P. E. (1973). Pattern classification and scene analysis. New York: Wiley.

Gold, J. M., Murray, R. F., Bennett, P. J., \& Sekuler, A. B. (2000). Deriving behavioral receptive fields for visually completed contours. Current Biology, 10, 663-666.

Graham, N. V. S. (1989). Visual pattern analyzers. New York: Oxford University Press.

Green, D. M., \& Swets, J. A. (1966). Signal detection theory and psychophysics. New York: Wiley.

Knill, D. C., \& Richards, W. (Eds.) (1996). Perception as Bayesian inference. New York: Cambridge University Press.

Medin, D. L., \& Schaffer, M. M. (1978). Context theory of classification learning. Psychological Review, 85, 207-238.

Minda, J. P., \& Smith, J. D. (2001). Prototypes in category learning: The effects of category size, category structure, and stimulus complexity. Journal of Experimental Psychology: Learning, Memory, \& Cognition, 27, 775-799.

Minda, J. P., \& Smith, J. D. (2002). Comparing prototype-based and exemplar-based accounts of category learning and attentional allocation. Journal of Experimental Psychology: Learning, Memory, \& Cognition, 28, 275-292.

Neri, P., Parker, A. J., \& Blakemore, C. (1999). Probing the human stereoscopic system with reverse correlation. Nature, 401, 695-698. 
Nosofsky, R. M. (1986). Attention, similarity, and the identificationcategorization relationship. Journal of Experimental Psychology: General, 115, 39-61.

Nosofsky, R. M. (1990). Relations between exemplar-similarity and likelihood models of classification. Journal of Mathematical Psychology, 34, 393-418.

RiNGaCh, D. L., SAPIRo, G., \& Shapley, R. (1997). A subspace reversecorrelation technique for the study of visual neurons. Vision Research, 37, 2455-2464.
Sekuler, A. B., Gaspar, C. M., Gold, J. M., \& Bennett, P. J. (2004). Inversion leads to quantitative, not qualitative, changes in face processing. Current Biology, 14, 391-396.

Tuan, B. S., Braje, W. L., Legge, G. E., \& Kersten, D. (1995). Human efficiency for recognizing 3-D objects in luminance noise. Vision Research, 35, 3053-3069.

(Manuscript received August 3, 2005;

revision accepted for publication January 9, 2006.) 